\title{
BiBLIOGRAPHY
}

1. M. L. Curtis and M. K. Fort, Jr., Homotopy groups of one-dimensional spaces, Proc. Amer. Math. Soc. vol. 8 (1957) pp. 577-579.

2. S. Eilenberg and S. MacLane, Relations between homology and homotopy groups of spaces, Ann. of Math. vol. 46 (1945) pp. 480-509.

3. M. K. Fort, Jr., Mappings of $S^{1}$ into one-dimensional spaces, Illinois J. Math. vol. 1 (1957) pp. 505-508.

4. H. B. Griffiths, Infinite products of semigroups and local connectivity, Proc. London Math. Soc. (1956) pp. 455-480.

UNIVERSITY OF GEORGIA

\section{ON ESSENTIAL FIXED POINTS}

\section{J. M. MARR}

The purpose of this note is to furnish an affirmative answer to a question posed at the Summer Institute on Set Theoretic Topology held at the University of Wisconsin in 1955. Let $X^{\boldsymbol{x}}$ denote the space of continuous functions of $X$ in to $X$ topologized by the compact open topology. A fixed point $p$ of a map $f \in X^{\boldsymbol{x}}$ is called essential if for each neighborhood $U$ of $p$ there is a neighborhood $N$ of $f$ such that if $g \in N$, then $g$ has a fixed point in $U$.

THEOREM. If $X$ is a compact Hausdorff space which has the fixed point property, then there is an $f \in X^{\boldsymbol{x}}$ such that each fixed point of $f$ is essential.

Proof. Let $x_{0}$ be any element of $X$, and consider the map $f \in X^{x}$ where $f(X)=x_{0}$. Let $U$ be any neighborhood of $x_{0}$. Then $N$ $=\{g: g(X) \subset U\}$ is a neighborhood of $f$ with the property that each $g \in N$ has a fixed point in $U$. Therefore $x_{0}$ is an essential fixed point of $f$. Since $x_{0}$ is the only fixed point of $f, f$ is the required map.

Kansas State College

Received by the editors June 16, 1958. 